\title{
Resource Allocation in Cellular Wireless Systems
}

\author{
Villy B. Iversen and Arne J. Glenstrup \\ Department of Telecommunication, Building 371 Technical University of Denmark, \\ DK-2800 Kongens Lyngby,Tel: (+45) 4525 3648, Fax: (+45) 45930355 \\ e-mail \{vbi,panic\}@tele.dtu.dk
}

Key words: Mobility, modelling, microcell, macrocell, UMTS

\begin{abstract}
In mobile communications an efficient utilisation of the channels is of great importance. In this paper we describe the basic principles for obtaining the maximum utilisation and study strategies for obtaining these limits. In general a high degree of sharing is efficient, but requires service protection mechanisms to guarantee the Quality of Service for all services. We study cellular systems with hierarchical cells, and the effect of overlapping cells, and we show that by call packing we obtain a very high utilisation. The models are generalisations of the Erlang-B formula, and include general arrival processes, and multi-rate (multi-media) traffic for third generation systems.
\end{abstract}

\section{INTRODUCTION}

The modelling of a cellular communication system is usually divided into modelling of (a) traffic, (b) structure, and (c) strategy. In comparison with plain old telephone systems the problems become more complex as e.g. the number of channels available depends on the position of the subscribers, the subscribers are moving, and the intelligence available for decision-making is very high.

\section{CELL DIMENSIONING}

We can model a basic cellular network of $N$ cells analytically by considering a state space in which each state is indexed by a vector $\left[i_{l}, \ldots, i_{N}\right]$ where $i_{k}$ is the number of customers being served by cell $k$. New calls, call terminations and call handovers are modelled by Poisson processes with state-dependent intensities, causing single state transitions. This model is generally non-trivial 
to solve, due to the handover transitions, but it has been shown that it can be approximated by a model in which the effect of handovers have been transformed into an increase in the birth and death rates [1,3]. This latter model, which has product form, can be solved and subsequently used to compute various performance measures (blocking probabilities for new calls and handovers, utilisation etc.). The approximation is not exact, but can be considered as a worst-case scenario when dimensioning the cells of a mobile network.

Measurements (e.g. [10]) have confirmed that new calls arrive according to a Poisson process with slow variations during the day. It has also been shown that handover traffic is more smooth than the Poisson process [9].

All the models considered in the following have product form and are insensitive to the holding time distribution. Because of the product form we may apply the convolution algorithm for loss systems, first published in [4]. In a network with direct routing we have product form between the routes. The convolution algorithm allows for class limitation by truncating the state space and for minimum allocation by aggregation of states [5]. The models are valid for any state-dependent Poisson process and multi-rate traffic. More details are given in [6].

\section{OVERLAY \& UNDERLAY STRUCTURE}

The performance of cellular mobile communication systems can be improved significantly by introducing macro cells. If a call (either a new or a hand over call) attempts to establish a connection in a micro cell but all the channels of that cell are busy, it may try to establish the connection in an overlaid macro cell. Figure 1 shows a two-level system with $\mathrm{N}$ microcells and one macrocell. Micro-cell number $i$ has $n_{i}$ channels of its own. The macrocell has a total of $m$ channels, but microcell number $i$ may at most borrow $m_{i}$ channels in the macrocell at a given point of time (class limitation). Thus the subscribers in a given microcell has a minimum allocation of $n_{i}$ channels and a maximum allocation of $n_{i}+m_{i}$. This allows us to guarantee a certain grade of service. The macro cell is common to a number of micro cells, i.e. it acts as a shared resource for a number of micro cells. The multi-layer structure is equivalent to that of a classical overflow system. Two call management strategies for operating macro cells exist [8]:

- Without rearrangement, i.e. once a call has established a connection in a channel in a macro cell, it continues to utilize the channel until the call is terminated.

- With rearrangement, if a call is using a channel in a macro cell it will rearrange to the micro cell (where the call is located) when a channel in that particular micro cell becomes idle. 
The rearrangement strategy increases the number of hand over operations and requires that the system keeps information about which micro cell a call belongs to.

The performance of multi-layer systems are calculated in the following way. Systems without rearrangement are similar to classical overflow systems and can be evaluated by well-known methods. If the rearrangement strategy is applied, the state transition diagram is reversible and blocking probabilities, utilisation, etc. are obtained using the reversibility, for instance by using the convolution algorithm [4].

\subsection{EXAMPLE}

We consider a network with 40 micro cells and one macro cell covering all the micro cells. Each micro cell has 40 channels. The number of channels in the macro cell is a variable. The termination rate of a call is one (the time unit is chosen as the average holding time) and the arrival rate of new calls is per time unit. A call in a micro cell tends to move to one of the neighbour cells with a constant rate of one. When changing cell the new cell is chosen among the neighbouring cells with equal probability. In order to reduce the blocking probability a number of channels is allocated to the macro cell.

Figure 2 features a plot of the total carried traffic versus the number of channels in the macro cell. If the call management strategy without rearrangement is applied the utilisation of the channels in the macro cell is close to one. If the rearrangement strategy is applied, the extra carried traffic per additional channel in the macro cell is higher than one erlang. This is due to the fact that when a call is blocked at the micro cell and gets a channel in the macro cell it will only remain in the macro cell until a channel in the micro cell is released. On average this time is only $1 / 40$ of a holding time (if only one call is waiting for a channel in the micro cell). In this way the shared resource (the macro channel) is made available for new calls as soon as possible. Obtaining the same amount of carried traffic (or correspondingly the same blocking probabilities) without rearrangement requires a significantly higher number of channels in the macro cell. However, the increased utilisation of the micro cells implies that a higher number of calls are blocked in the micro cells but accepted in the macro cell. Thus the total number of rearrangements increases. If the number of channels in the macro cell becomes large the system starts to make unnecessary rearrangements. A rearrangement of a call from the macro cell to a micro cell $\mathrm{k}$ is unnecessary if no other micro cell requests to use the released channel in the macro cell. When the number of channels in the macro cell is large the utilisation is small and many unnecessary rearrangement occur. 


\section{OVERLAPPING CELL BOUNDARIES}

Above, we considered one macrocell overlapping all microcells. We may also have overlap between the microcells. In Figure 3 subscribers in area 1 have access to $n_{1}$ channels, subscribers in area 2 access to $n_{2}$ channels, and subscribers in area 12 have access to $n_{1}+n_{2}$ channels. Therefore, subscribers in area 12 will experience a smaller blocking probability than subscribers in the other areas. In an intelligent system we can freely hand-over calls in area 12 between the two base stations. macrocell to the microcell when a channel

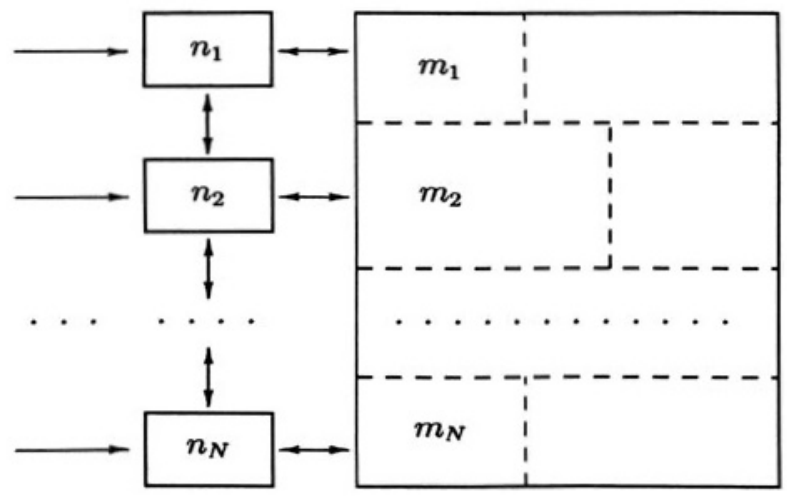

Figure I Model of a cellular system with $\mathrm{N}$ microcells and one macrocell corresponding to a link model offered more traffic streams with minimum and maximum The model has product form.

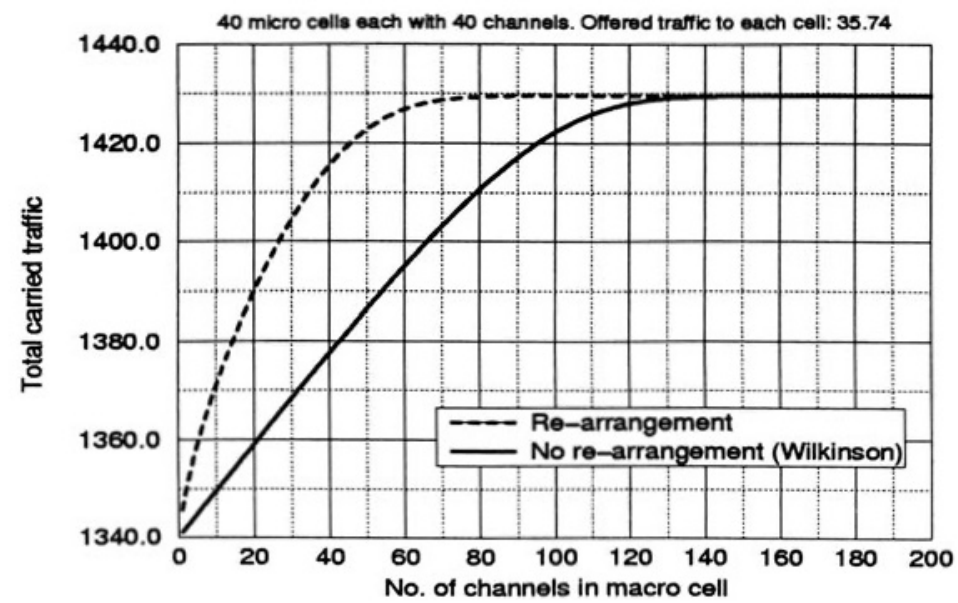

Figure 2 The total carried traffic as a function of the number of channels in the macro cell. By adding 20 channels in the macro cell we notice that the total carried traffic increases by 50 erlang. 


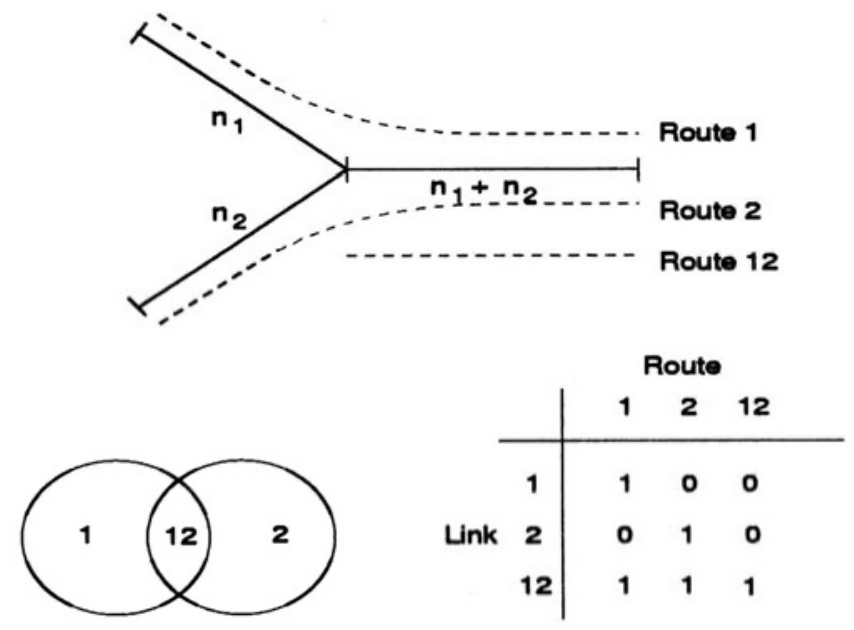

Figure 3 Example of two overlapping cells and the equivalent circuit-switched network with direct routing.

becomes available. Denoting the number of existing connections in the area $i$ by $x_{i}$ we notice that we have the following restrictions:

$$
\begin{array}{rrrr} 
& 0 & \mathrm{x}_{1} & \mathrm{n}_{1} \\
0 & \mathrm{x}_{2} & \mathrm{n}_{2} \\
& 0 & \mathrm{x}_{1}+\mathrm{x}_{2}+\mathrm{x}_{12} & \mathrm{n}_{1}+\mathrm{n}_{2}
\end{array}
$$

This is equivalent to the circuit switched communication network with direct routing shown in the Figure 3. We describe a network with direct routing by the link, the routes and the number of channels $c_{i j}$ (element of the matrix) a route requires on each link. The models are valid for multi-slot systems with individual slot size on each link.

We notice that if all channels are busy in e.g. area 1, then we may hand-over a connection in the area 12 from base station one to base station two. Thus we assume optimal rearrangement (call packing). In a multi-cell systems this rearrangement may be necessary at several levels. Thus we assume that the system has global optimal intelligence. In Figure 4 we consider a system with three cells, which are mutually overlapping, so that subscribers in some areas may have access to two base stations, but not three. Therefore, subscribers in overlapping areas will experience a smaller blocking probability than subscribers in the separate areas. In an intelligent system with optimal packing we can rearrange calls in the overlapping areas from one base station to another. Thus we assume that the system has global optimal intelligence. The model with restrictions on the number of simultaneous calls is equivalent to a circuit switched communication network with direct routing [5] as shown in the Table 


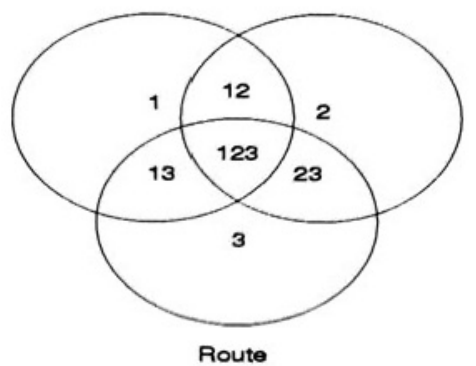

\begin{tabular}{r|rrrrrrr} 
& 1 & 2 & 3 & 12 & 13 & 23 & 123 \\
\hline 1 & 1 & 0 & 0 & 0 & 0 & 0 & 0 \\
2 & 0 & 1 & 0 & 0 & 0 & 0 & 0 \\
3 & 0 & 0 & 1 & 0 & 0 & 0 & 0 \\
12 & 1 & 1 & 0 & 1 & 0 & 0 & 0 \\
13 & 1 & 0 & 1 & 0 & 1 & 0 & 0 \\
23 & 0 & 1 & 1 & 0 & 0 & 1 & 0 \\
123 & 1 & 1 & 1 & 1 & 1 & 1 & 1
\end{tabular}

Figure 4 Example of three overlapping cells and the corresponding equivalent circuitswitched network with direct routing.

of Figure 4. A link corresponds to a restriction The number of routes becomes equal to the number of separate areas, whereas the number of links becomes equal to the number of restrictions, which is equal to the number connected areas (paths) we can built up from the distinguishable areas. If all $\mathrm{N}$ cells are overlapping the number of links become equal to $2^{\mathrm{N}} \quad 1$, as we exclude the empty set. For the case considered in Figure 4 the number of routes becomes 6 and the number of links becomes 7 (in fact, one of the restrictions is superfluous).

For the case considered the carried traffic as a function of the overlapping is shown in Figure 5. We notice, that we have the same capacity as for full availability when the overlapping is greater than $20 \%$. This will be the case in most real systems. The model with overlay cell is included in this model has the overlay cell is a cell overlapping all other cells.

Evaluation methods: For small networks we have exact algorithms for evaluating the end to $\mathrm{cnd}$ blocking probability for each route, i.c. for each area. The convolution algorithm [4] allows calculation of both time congestion, call congestion, and traffic congestion for Multi-slot Binomial - Poisson - Pascal traffic.

As the number of routes and links for realistic systems (e.g, GSM) becomes very large, the exact methods are not applicable. Then numerical simulation and approximate methods as the Erlang reduced load (Erlang fixed point) methods has to be used. However for large networks, where a typical route 


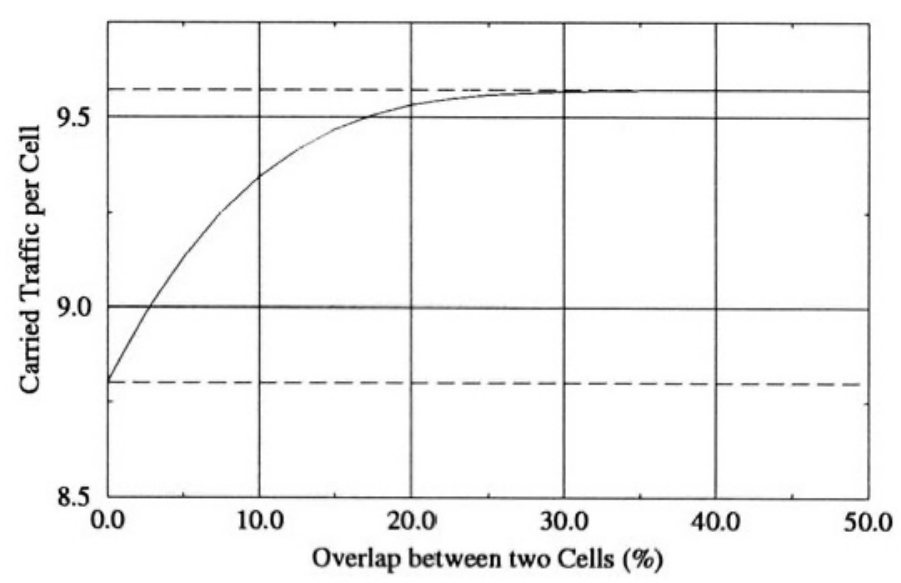

Figure 5 System with three cells adjacent to each other. $\mathrm{A}=10$ erlang per cell, $\mathrm{n}=12$ channels per cell. $10 \%$ overlap means that a cell has $10 \%$ overlap with both of the two other cells, but there is no overlap between all three cells.

may use 20 links, these methods are not very good.

Decentralized intelligence. In the above models we have assumed that the call packing is optimal. We may thus implement many successive rearrangements to move one idle channel from one cell to another. In e.g. DECT systems the intelligence is distributed to the individual handsets. Thus it is possible to let the handset, which knows the state of the channels at the local base station in the area, make local decisions based on the local information. Thus a handset may hand-over a call from a base station with all channels busy to a base station with idle channels. However, this strategy will not always be globally optimal (but we know the optimal reference value). A random model will choose an idle channel at random. These models can (only) be evaluated by simulation.

\section{UMTS}

Until this point, we have been considering 2nd generation mobile systems, i.e. the GSM and DECT systems. However, in the near future we will sec the introduction of the 3rd generation mobile systems, UMTS (Universal Mobile Telecommunications System). The main cxtension in UMTS is the introduction of packet-switches connections for data transfer, and a design that is able to supply a greater bandwidth than GSM, typically $384 \mathrm{Kbps}$, and up to 2 Mbps. However, the increased bandwidth is not evenly distributed: the radio interface is designed so that the available capacity decreases with the distance of the mobile terminal from the base station. High-speed data transfer requires 
the largest bandwidth, and a voice connection the least, so to prevent a single terminal far from the base station using up all the capacity, connections there will be restricted to low bandwidths. Thus we can model a UMTS cell as a number of concentric rings with different available services, as shown in Figure 6 [2]. The packet switched services may be transformed to circuit switched traffic by using effective or equivalent bandwidths [7].

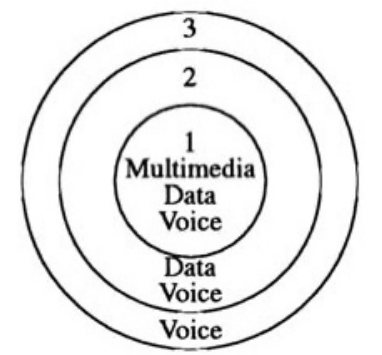

$\begin{array}{ll}\text { Service } & \text { Bandwidth } \\ \text { Multimedia } & 2 \mathrm{Mbps} \\ \text { Data } & 384 \mathrm{Kbps} \\ \text { Voice } & 16 \mathrm{Kbps}\end{array}$

Figure 6 An example of a UMTS base station: in the outer rings only the low-bandwidth services are available

\subsection{TRAFFIC MODELLING RESULTS}

We model a UMTS cell carrying 20 channels in total, with only 10 and 5 being available in ring 2 and 3 . Each call requires 1,2 or 5 channels for a voice, data or multimedia connection, respectively. Table 1 shows the blocking probabilities for the services and the total carried traffic in the cell when more channels are available in the outer rings (10-13 in ring 2, and 5-8 in ring 3 ).

Normally, however, the number of channels available in the rings cannot change, and with the basic setup of $20 / 10 / 5$ available channels, this causes voice call blocking in ring 3 to be 4 times as high as voice calls in ring 1 . If we want to even out the blocking, we can enforce a reservation strategy whereby a number of "private" channels are reserved exclusively for calls in ring 2 and 3. Table 2 shows how blocking and carried traffic change under this strategy. Note that reserving one channel in each outer ring results in an increase in the total carried traffic; this is due to a carried traffic increase in ring 2 greater than the carried traffic decrease in ring 1. Figure 7 is a plot of how the blocking depends on the number of reserved channels in the outer rings.

\section{CONCLUSIONS}

By exploiting the capabilities of digital systems we are able to obtain a high utilisation of the radio channels. In particular systems with over-lapping cells and overlaid cells are able to manage local overload and at the same time guarantee a certain grade-of-service. If we are able to rearrange calls, then we are 


\begin{tabular}{|c|c|c|c|c|c|c|c|}
\hline & \multicolumn{3}{|c|}{ Ring 1} & \multicolumn{2}{|c|}{ Ring 2} & Ring 3 & \multirow{4}{*}{$\begin{array}{l}\text { Carried } \\
\text { traffic }\end{array}$} \\
\hline & M-media & Data & Voice & Data & Voice & Voice & \\
\hline \multirow{2}{*}{$\begin{array}{l}\text { Offered traffic } \\
\text { Channels/call }\end{array}$} & 0.5 & 1 & 2 & 1 & 2 & 2 & \\
\hline & 5 & 2 & 1 & 2 & 1 & 1 & \\
\hline \multirow{2}{*}{$\begin{array}{l}\text { Max channels } \\
\text { Blocking }\end{array}$} & \multicolumn{3}{|c|}{20} & \multicolumn{2}{|c|}{10} & 5 & \multirow[t]{2}{*}{11.350085} \\
\hline & 0.176014 & 0.047955 & 0.020968 & 0.14304 & 0.060094 & 0.082878 & \\
\hline \multirow{2}{*}{$\begin{array}{l}\text { Max channels } \\
\text { Blocking }\end{array}$} & \multicolumn{3}{|c|}{20} & \multicolumn{2}{|c|}{11} & 6 & \multirow[t]{2}{*}{11.476073} \\
\hline & 0.185510 & 0.051442 & 0.022568 & 0.10826 & 0.045381 & 0.052423 & \\
\hline \multirow{2}{*}{$\begin{array}{l}\text { Max channels } \\
\text { Blocking }\end{array}$} & \multicolumn{3}{|c|}{20} & \multicolumn{2}{|c|}{12} & 7 & \multirow[t]{2}{*}{11.548188} \\
\hline & 0.191657 & 0.053890 & 0.023737 & $0.08498^{\prime}$ & 0.035924 & 0.037797 & \\
\hline \multirow{2}{*}{$\begin{array}{l}\text { Max channels } \\
\text { Blocking }\end{array}$} & \multicolumn{3}{|c|}{20} & \multirow{2}{*}{\multicolumn{2}{|c|}{$\frac{13}{0.0710450}$}} & 8 & \multirow[t]{2}{*}{11.586782} \\
\hline & 0.195412 & 0.055411 & 0.024497 & & & 0.030911 & \\
\hline
\end{tabular}

Table 1 Blocking probabilities and carried traffic under four different channel assignments

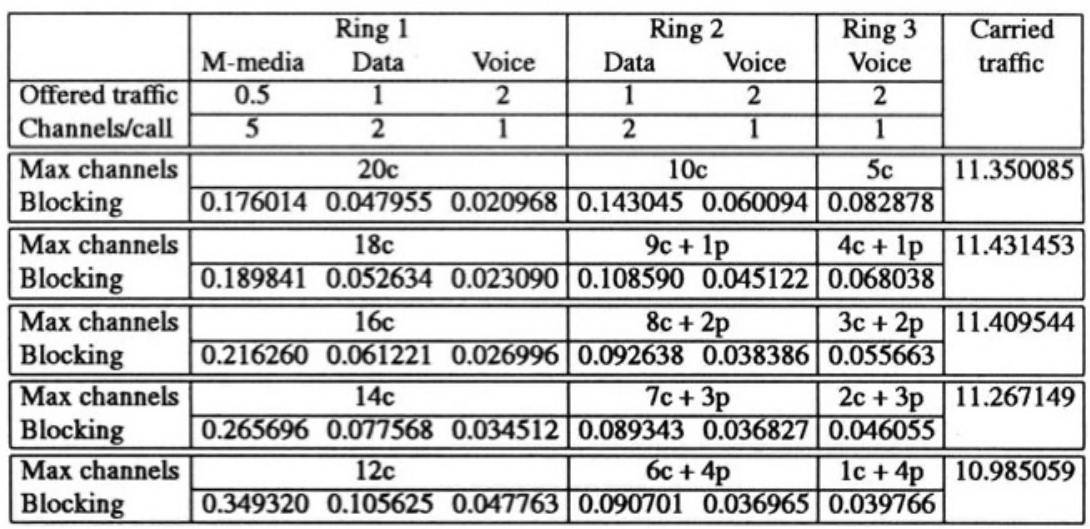

Table 2 Blocking probabilities and carried traffic under five different partitions of 20 channels into (c)ommon channels and (p)rivate channels reserved for a specific ring

able to evaluate the systems. For systems with fixed slot-assignment the exact solution is based on a very large number of linear equations. For multi-rate systems we have to protect wide-band traffic by using trunk reservation or class limitation.

\section{References}

[1] Christiansen, C. \& Iversen, V.B. \& Nasr, S. (1993): Product forn solutions for cellular mobile communication systems. NTS 11,11 th Nordic Teletraffic Seminar, Stockholm 1993. 8 pp.

[2] Dekocker, S. (1999): Traffic problems in cellular wideband systems. Master's thesis. Dpt. of Telecommunication, Technical University of Denmark. 98 pp. 


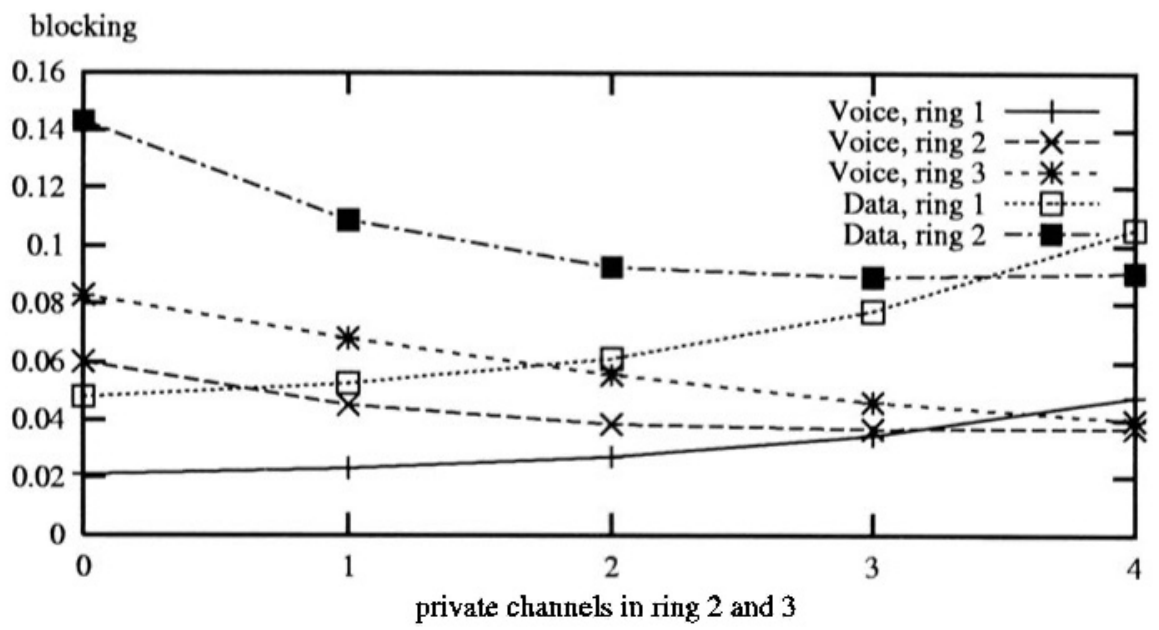

Figure 7 Blocking as a function of the number of private channels in the outer rings

[3] Everitt, D. (1991): Product Form Solutions in Cellular Mobile Communication Systems, Teletraffic and Datatraffic in a Period of Change, ITC-13, A. Jensen \& V. B. Iversen (editors), North Holland, 1991, pp. 483-488.

[4] Iversen, V. B. (1987): The Exact Evaluation of Multi-Service Loss Systems with Access Control. Teleteknik, English ed., Vol. 31 (1987): 2, $56-61$.

[5] Iversen, V. B. (1995): Traffic Engineering of Cellular Mobile Communication Systems. ITC Regional Seminar in Bangkok, November 28 December 1, 1995. $10 \mathrm{pp}$.

[6] Iversen, V.B. (2000): Teletraffic Engineering. Chapter 11: Multi-dimensional loss systems. $61 \mathrm{pp}$. Dpt. of Telecommunications, Technical University of Denmark, 2000.

[7] Kelly, F. (1995): Notes on effective bandwidthsr, pp. 141-168 in Stochastic networks, theory and applications. Royal Statistical Society, London 1995.

[8] Lagrange, X. (1997): Multitier cell design. IEEE Communications Magazine, August 1997, pp 60-64.

[9] Rajaratnam, M. \& Takawira, F. (1999): Non-classical traffic modelling and performance analysis of cellular mobile networks with and without channel reservation. University of Natal, Durban, South Africa. $39 \mathrm{pp}$. To appear in IEEE Trans. on Vehicular Technology.

[10] Smith, P.J. \& Sathyendran, A. \& Murch, A.R. (1999): Analysis of traffic distribution in cellular networks. 49th IEEE Vehicular Technology Conference, May 16-129, 1999, Houston, Texas, USA. Vol. 3, pp. 20752079. 